\title{
Frailty: joining the giants
}

\author{
Peter Crome MD DSC, Frank Lally PhD
}

See related research article by Rockwood and colleagues at www.cmaj.ca/cgi/doi/10.1503/cmaj.101271.

The giants of geriatrics are immobility, instability, incontinence and intellectual impairment. They have in common multiple causation, chronic course, deprivation of independence and no simple cure.

- Bernard Isaacs, The Challenge of Geriatric Medicine, Oxford University Press, 1997

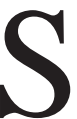

ince Isaacs made that statement, further giants of geriatric medicine, such as iatrogenesis, have been added to the list. The concept of geriatric syndromes has been universally accepted with internationally agreed-upon definitions and numerous guidelines for assessment and treatment. The last decade has seen a burgeoning interest in yet another giant: the frailty syndrome.

Frailty can be thought of as a combination of factors that influence a person's physiologic state to the extent that function is greatly reduced and the person becomes more vulnerable to external stressors. This vulnerability can result in disability, admission to hospital or a long-term care facility and increased risk of death.

Although the concept of frailty is generally accepted (despite the pejorative connotations of the term "frail"), its definition is not. Separate models by Fried and Rockwood are often cited and have been employed in clinical trials.

In Fried's model, the presence of three or more of five indicators (unintended weight loss, tiredness, weak grip strength, slow walking speed and physical inactivity) predicts a range of adverse outcomes including falls, disability, admission to hospital and death. ${ }^{1}$ Fried and colleagues stated that frailty was distinct from comorbidity and disability, although the categories did overlap. Although the "diagnosis" of frailty using defined cutoff values is attractive, the Fried criteria are difficult to apply to all groups, such as those patients who are acutely ill and the most dependent. ${ }^{2}$

Various amendments to the Fried criteria have been suggested by other investigators. A fairly recent study attempted to produce an instrument for identifying frailty among patients at primary care institutions in Europe, which the authors claimed was a valid alternative to the Fried model. ${ }^{3}$ The Fried criteria do not specifi- cally include direct measurements of mental health or psychosocial status, measures that clinicians $^{4}$ and older people ${ }^{5}$ alike feel are an important part of the definition of frailty. In fact, more recently, Fried and coauthors suggested that these factors were related to frailty. ${ }^{6}$

An alternative model that took a quantitative approach and defined frailty as the accumulation of deficits was suggested by Rockwood and coworkers. ${ }^{7,8}$ They proposed that the risk of becoming frail increased with certain deficits. These deficits may be multisystem physiologic or cognitive changes (not necessarily diseases) that can be seen in clinical data. The deficits exist on a continuous scale and can be indexed. Thus, the more deficits that are present, the higher the index and the greater the risk that the patient will become frail.

Unlike the Fried criteria, the Rockwood model can incorporate the patient's mental health or psychosocial status. Rockwood has further developed the concept of accumulated deficit in a paper published in this issue of $C M A J$, in which he showed that the number of deficits predicted mortality across the adult lifespan, although the prevalence of frailty was much lower in younger patients. ${ }^{9}$ However, this model has not been fully accepted in the clinical setting, possibly because of its complexity. Rockwood and colleagues tried to address this problem by introducing a sevenpoint clinical scale for measuring frailty..$^{10}$ This scale is judgment-based and asks the clinician to evaluate a patient's fitness, wellness, comorbidity and three levels of frailty based on dependency.

Evidence that these two approaches may share certain similarities comes from a further analysis of the Womens' Health and Aging Studies. These prospective, observational studies

\section{KEY POINTS}

- Frailty is a combination of factors influencing a person's physiologic state such that he or she may become more vulnerable to external stressors.

- Although the concept of frailty is generally accepted, its definition is not.

- Rockwood's model for assessing frailty proposes that certain deficits, including those in areas of mental health or psychosocial issues, increase a person's risk of becoming frail. 
showed that the number of abnormal physiologic systems (as indicated by the measurement of biomarkers such as hemoglobin or markers of inflammation) was associated with an increased risk of frailty in the same way as Rockwood's accumulated deficits or Fried's criteria. ${ }^{11}$

Although Fried's and Rockwood's models have both been validated, neither of them has been incorporated into routine clinical practice. Indeed, Rockwood and colleagues themselves suggested that it may be too early to select one model over the other; instead, they recommend that both models be explored to further our understanding of the concept of frailty. ${ }^{12}$ A study based on the Cardiovascular Health Study using a deficit index and a phenotypic frailty index ${ }^{13}$ concluded that, although the deficit index was better at identifying increased risk of death, an integration of both indices may increase the precision of such a prediction among vulnerable older people.

Calculating the prevalence of frailty within a population is important for providing and financing hospital and social care. However, the value of identifying an individual patient as being frail is less clear - any medical intervention will depend on identifying specific causal factors. An exception to this may be encouraging patients to exercise, which has proven beneficial in many studies of older people with and without frailty. ${ }^{14}$

Identifying frailty may be of clinical value when determining risk for patients having elective surgery. Makary and coauthors ${ }^{15}$ showed that frailty, as determined by the Fried criteria, predicted postoperative complications, length of stay in hospital and discharge from hospital to a skilled or assisted-living facility. Frailty also improved the predictive power of other scales used for preoperative assessment (e.g., Lee, Eagle and the American Society of Anesthesiologists). Further studies in different settings should clarify the utility of preoperative screening for frailty.

Geriatricians have long argued for the inclusion of patients with frailty in clinical trials. For this reason, an agreement on a definition of frailty would be helpful. Although reaching such an agreement may be difficult, a consensus statement on sarcopenia has been written, the definition of which includes two of the five Fried criteria. ${ }^{16}$

Clearly, exercise is an effective approach to combating frailty in a wide variety of settings. ${ }^{13}$ However, individualized approaches are also needed. Cost-effective preventive and therapeutic interventions will be necessary for the increasingly aging population expected over the next 25 years, which is a good reason for continued research on frailty and its causes.

\section{References}

1. Fried LP, Tangen CM, Walston J, et al. Frailty in older adults: evidence for a phenotype. J Gerontol A Biol Sci Med Sci 2001; 56:M146-56.

2. Hubbard RE. O'Mahony MS, Woodhouse KW. Characterising frailty in the clinical setting: a comparison of different approaches. Age Ageing 2009:38:115-9.

3. Romero-Ortuno R, Walsh CD, Lawlor BA, et al. A frailty instrument for primary care: findings from the Survey of Health, Ageing and Retirement in Europe (SHARE). BMC Geriatr 2010;10:57.

4. Fillit H, Butler RN. The frailty identity crisis. J Am Geriatr Soc 2009;57:348-52.

5. Puts MTE, Shekary N, Widdershoven $\mathrm{G}$ et al. The meaning of frailty according to Dutch older frain and non-frail persons. $J$ Aging Stud 2009;23:258-66

6. Szanton SL, Seplaki CL, Torpe RJ, et al. Socioeconomic status is associated with frailty: the Women's Health and Aging Studies. J Epidemiol Community Health 2010;64:63-7.

7. Mitnitski AB, Mogilner AJ, Rockwood K. Accumulation of deficits as a proxy measure of aging. ScientificWorldJournal 2001;1:323-36.

8. Rockwood K, Mitniski A. Frailty in relation to the accumulation of deficits. J Gerontol A Biol Sci Med Sci 2007;62:722-7.

9. Rockwood K, Song X, Mitnitski A. Changes in relative fitness and frailty across the adult lifespan: evidence from the Canadian National Population Health Surveys. CMAJ 2011;183:E487-94.

10. Rockwood K, Song X, MacKnight C, et al. A global clinical measure of fitness and frailty in elderly people. CMAJ 2005;173: 489-95.

11. Fried LP, Xue QL, Cappola AR, et al. Nonlinear multisystem physiological dysregulation associated with frailty in older women: implications for etiology and treatment. J Geront A Biol Sci Med Sci 2009;64:1049-57.

12. Rockwood K, Andrew M, Mitnitski A. A comparison of two approaches to measuring frailty in elderly people. $J$ Gerontol A Biol Sci Med Sci 2007;62:738-43.

13. Kulminski AM, Ukraintseva SV, Kulminskaya IV, et al. Cumulative deficits better characterize susceptibility to death in elderly people than phenotypic frailty: lessons from the Cardiovascular Health Study. J Am Geriatr Soc 2008;56:898-903.

14. Souto Barreto P. Exercise and health in frail elderly people: a review of randomized controlled trials. Eur Rev Aging Phys Act 2009;6:75-87.

15. Makary MA, Segev DL, Pronovost PJ, et al. Frailty as a predictor of surgical outcomes in older patients. J Am Coll Surg 2010; 210:901-8.

16. Cruz-Jentoft AJ, Baeyens JP, Bauer JM, et al. Sarcopenia: European consensus on definition and diagnosis. Age Ageing 2010; 39:412-23.

Affiliations: Peter Crome is with Keele University and North Staffordshire Combined Healthcare National Health Service Trust, Keele, UK, and Frank Lally is with the Institute for Science and Technology in Medicine, Keele University, Keele, UK.

Acknowledgements: The authors thank Ruth Hubbard and Avan Ahie Sayer for useful conversations in the preparation of this paper. 